\title{
Hepatic pathology may develop before the Fontan operation in children with functional single ventricle: An autopsy study
}

\author{
Matthew C. Schwartz, MD, ${ }^{\mathrm{a}}$ Lisa Sullivan, MD, ${ }^{\mathrm{b}}$ Meryl S. Cohen, MD, ${ }^{\mathrm{a}}$ Pierre Russo, MD, ${ }^{\mathrm{b}}$ \\ Anitha S. John, MD, PhD, ${ }^{\mathrm{a}, \mathrm{e}}$ Rong Guo, MS, ${ }^{\mathrm{c}}$ Marta Guttenberg, MD, ${ }^{\mathrm{b}}$ and Elizabeth B. Rand, MD ${ }^{\mathrm{d}}$
}

\begin{abstract}
Objective: Liver fibrosis has emerged as an important long-term complication of the Fontan operation. We aimed to describe liver histology at autopsy in patients who had undergone the Fontan operation and to determine whether patient variables are associated with the degree of fibrosis.
\end{abstract}

\begin{abstract}
Methods: A review was performed of all patients with a history of the Fontan operation who died and underwent autopsy at our institution from 1980 to 2009. Autopsy liver slides were evaluated independently by 2 pathologists.

Results: Twenty-two patients were studied. The median interval between Fontan and death was 20 days (range, 1 day-17.5 years). Portal fibrosis was observed in 20 (91\%) patients and sinusoidal fibrosis was observed in 17 $(77 \%)$ patients. Using simple linear regression, time from the Fontan operation was significantly associated with the degree of portal fibrosis on Ishak $(P=.03)$ and modified Scheuer fibrosis $(P=.02)$ scales. Significant portal fibrosis was observed in $8(57 \%)$ of the 14 patients who died 30 days or less after the Fontan operation. In these 14 patients, severity of portal fibrosis was associated with length of hospitalization after pre-Fontan cardiac operations $(P=.03)$ and pre-Fontan mean right atrial pressure $(P=.04)$.

Conclusions: At autopsy, hepatic fibrosis was commonly observed in patients who had undergone the Fontan operation. Portal fibrosis has been previously unrecognized in this population. Significant portal fibrosis occurred in most who died soon after the Fontan procedure and was associated with pre-Fontan morbidity. Hepatic disease in the single-ventricle population is multifactorial and may begin before the Fontan operation. (J Thorac Cardiovasc Surg 2012;143:904-9)
\end{abstract}

The Fontan operation was first described in 1971 and has become a widely used palliation for patients with singleventricle congenital heart disease. ${ }^{1}$ Over the past 40 years, the Fontan operation has undergone several modifications, but its basic principle remains constant: blood flows passively through the pulmonary vascular bed as a total cavopulmonary connection routes systemic venous return directly to the pulmonary arterial circulation.

Survival after the Fontan procedure has improved over the past 4 decades; however, complications of the abnormal circulation have developed, including liver fibrosis and cirrhosis. $^{2-4}$ Hepatic fibrosis was first described in this population in 1981 and is now recognized by cardiologists and hepatologists as an important long-term complication of the palliative strategy, which may shorten life

From the Divisions of Cardiology ${ }^{\mathrm{a}}$ and Pathology, ${ }^{\mathrm{b}}$ Biostatistics and Data Management Core, ${ }^{\mathrm{c}}$ and the Division of Gastroenterology, ${ }^{\mathrm{d}}$ The Children's Hospital of Philadelphia, Philadelphia, Pa; and the Division of Cardiology, ${ }^{\mathrm{e}}$ Children's National Medical Center, George Washington University, Washington, DC.

Disclosures: Authors have nothing to disclose with regard to commercial support.

Received for publication May 12, 2011; revisions received July 30, 2011; accepted for publication Aug 25, 2011; available ahead of print Sept 28, 2011.

Address for reprints: Matthew C. Schwartz, MD, Division of Cardiology, The Children's Hospital of Philadelphia, Room 8NW25, 34th St and Civic Center Blvd, Philadelphia, PA 19103 (E-mail: schwartzm@email.chop.edu).

0022-5223/\$36.00

Copyright (c) 2012 by The American Association for Thoracic Surgery doi:10.1016/j.jtcvs.2011.08.038 expectancy. ${ }^{5}$ Liver cirrhosis is associated with significant morbidity including hepatocellular carcinoma and may preclude cardiac transplantation, a therapeutic option for some patients. $^{6}$

Few reports exist that describe pathologic changes in liver histology in patients who have undergone the Fontan operation. Indeed, risk factors for the development of hepatic fibrosis have not been clearly identified. ${ }^{6-8}$ Moreover, the liver fibrosis that has been previously reported has been centrilobular, affecting the area around the central vein and extending outward along the sinusoids (sinusoidal fibrosis). Portal-based fibrosis, as typically seen in inflammatory hepatopathies, has not been observed in patients who have had the Fontan operation..$^{6-8}$ We aimed to characterize hepatic histology in children and adolescents who died after the Fontan operation and to determine whether demographic, anatomic, surgical, temporal, and clinical variables were associated with the degree of observed hepatic fibrosis in these patients.

\section{METHODS}

\section{Patients}

The study protocol was approved by the Institutional Review Board at the Children's Hospital of Philadelphia. We retrospectively identified all patients who underwent the Fontan operation, died, and subsequently underwent autopsy at the Children's Hospital of Philadelphia between January 1980 and December 2009. Patients were included if the autopsy 
included evaluation of the liver and if the original histologic blocks were available. Patients with another known cause of liver disease such as viral hepatitis were excluded.

The medical history of each patient was reviewed, and demographic, anatomic, surgical, temporal, and clinical variables were extracted. Because all included patients underwent autopsy, the cardiac specimen of each patient was reviewed to confirm intracardiac anatomy and Fontan type.

\section{Histologic Grading}

Paraffin-embedded, 10\% neutral buffered formalin-fixed, 4-um-thick sections were obtained from each autopsy block that included sections of liver tissue. Each slide was deparaffinized with xylene and stained with hematoxylin and eosin, reticulin, Gomori trichrome, or reticulin. Each slide was then reviewed independently by 2 pathologists (P.R., L.S.) who were blinded to all patient variables. Histologic characteristics were graded semiquantitatively as summarized in Appendix 1. Portal fibrosis was graded using 2 staging systems that have been used extensively in patients with chronic inflammatory hepatopathies, the Ishak fibrosis stage and the modified Scheuer fibrosis stage. ${ }^{9-11}$ Hepatic sinusoidal fibrosis was graded using an adaptation of a scale previously published ${ }^{8}$ and portal inflammation was graded. If the 2 pathologists scored a feature of the specimen differently, then they conferred to arrive at a final score.

\section{Statistical Analysis}

Summary statistics are reported as median with range or number of patients with percentage. Simple linear regression was used to determine whether patient variables were significantly associated with histologic grading results. Inasmuch as histologic grades are ordinal, the more sophisticated cumulative logit model was also conducted to confirm the results of simple linear regression.

\section{RESULTS}

\section{Patient Characteristics}

Twenty-two patients met inclusion criteria and their baseline characteristics are summarized in Table 1. Sixteen $(72 \%)$ patients had hypoplastic left heart syndrome, 3 $(14 \%)$ had tricuspid atresia, and $3(14 \%)$ had other

TABLE 1. Baseline characteristics of 22 patients with Fontan circulation

\begin{tabular}{lc}
\hline & Result* \\
\hline Demographic & \\
Sex, male & $14(64 \%)$ \\
Age at Fontan operation (y) & $1.62(0.86-5.75)$ \\
Age at death (y) & $1.97(0.93-23.25)$ \\
Fontan-to-death interval & $20 \mathrm{ds}(1 \mathrm{~d}-17.5 \mathrm{y})$ \\
Year Fontan operation performed & $1989(1985-2004)$ \\
Anatomic & \\
Hypoplastic left heart syndrome & $16(72 \%)$ \\
Tricuspid atresia & $3(14 \%)$ \\
Other & $3(14 \%)$ \\
Surgical & \\
Type of Fontan operation & \\
Lateral tunnel & $15(68 \%)$ \\
Extracardiac & $2(9 \%)$ \\
Atriopulmonary & $2(9 \%)$ \\
Other & $3(14 \%)$ \\
Fenestrated Fontan & $6(27 \%)$ \\
\hline
\end{tabular}

*Expressed as median with range or as number. single-ventricle malformations. Fifteen $(68 \%)$ patients underwent a lateral tunnel Fontan, $2(9 \%)$ an extracardiac Fontan, $2(9 \%)$ an atriopulmonary Fontan, $2(9 \%)$ had an intra-atrial tube graft placed from the inferior vena cava to the right pulmonary artery, and 1 a right atrium-right ventricle Fontan connection. Six (27\%) of the Fontan baffles were fenestrated. The median age at the time of Fontan procedure was 1.62 years (range, $0.86-5.75$ years) and the median interval between Fontan operation and death was 20 days (range, 1 day- 17.5 years). The median age at death was 1.97 years (range, 0.93-23.25 years). The Fontan operations were performed from 1985 to 2004 and the median year of operation was 1989 .

The most common cause of death was cardiac failure (Table 2). Three patients underwent cardiac transplantation for failing Fontan physiology. One patient (No. 19) died during the transplantation, and postmortem cultures grew Staphylococcus pneumoniae and Staphylococcus aureus, another patient (no. 22) died 2 days after transplantation of postoperative bleeding, and another (no. 20) died 4 months after heart transplant with ongoing graft rejection, cytomegalovirus gastritis, and acute cerebral hemorrhage. Hepatitis $\mathrm{C}$ status was only documented in 3 of the patients (nos. 19, 21, and 22), all of whom were negative. The 3 longest surviving patients (nos. 20-22) had a history of proteinlosing enteropathy.

\section{Histologic Findings and Analysis}

Histologic grading of portal fibrosis and sinusoidal fibrosis for each patient is listed in Table 2 . Twenty $(90 \%)$ patients had at least some degree of portal fibrosis by Ishak or modified Scheuer scales. Significant portal fibrosis was common; $9(41 \%)$ patients had Ishak stage 3 or greater and $9(41 \%)$ patients had modified Scheuer stage 2 or greater. Figure 1, $A$, shows Ishak stage 4 and modified Scheuer stage 3 fibrosis in a 20.1-year-old patient with tricuspid atresia who died 16.5 years after undergoing atriopulmonary Fontan. Portal-based cirrhosis (Ishak stage 6 or modified Scheuer stage 4) was uncommon and found in only 1 patient, shown in Figure 1, $B$, who had hypoplastic left heart syndrome and died 4.0 years after lateral tunnel Fontan.

Of note, portal fibrosis was common in those who died within 1 month of the Fontan operation because 12 of 14 $(86 \%)$ patients showed at least some degree of fibrosis by Ishak or modified Scheuer scales. Figure $1, C$, shows stage 4 Ishak fibrosis in a 2-year-old child with hypoplastic left heart syndrome who died 1 day after a lateral tunnel Fontan procedure.

At least some degree of sinusoidal fibrosis was found in $17(77 \%)$ patients (Table 2). Of the 5 patients who had no sinusoidal fibrosis, 4 died 20 days or less after the Fontan operation. Ten of the 14 patients who died within 1 month of Fontan had at least some degree of sinusoidal fibrosis, but only 2 had sinusoidal fibrosis greater than stage 1 . 
TABLE 2. Histologic liver findings at autopsy in 22 Fontan patients

\begin{tabular}{|c|c|c|c|c|c|c|c|c|c|}
\hline $\begin{array}{l}\text { Pt } \\
\text { no. }\end{array}$ & $\begin{array}{c}\text { Age at } \\
\text { death }(y)\end{array}$ & $\begin{array}{c}\text { Death } \\
(\mathbf{y})\end{array}$ & $\begin{array}{c}\text { Fontan to } \\
\text { death interval }\end{array}$ & Anatomy & $\begin{array}{c}\text { Fontan } \\
\text { type }\end{array}$ & $\begin{array}{c}\text { Ishak } \\
\text { fibrosis } \\
\text { stage (0-6) }\end{array}$ & $\begin{array}{c}\text { Scheuer } \\
\text { fibrosis } \\
\text { stage }(0-4)\end{array}$ & $\begin{array}{c}\text { Sinusoidal } \\
\text { fibrosis } \\
\text { score }(0-4)\end{array}$ & Cause of death \\
\hline 1 & 1.28 & 1987 & $1 \mathrm{~d}$ & HLHS & LT & 0 & 0 & 1 & Cardiac failure \\
\hline 2 & 2.04 & 1988 & $1 \mathrm{~d}$ & HLHS & LT & 4 & 2 & 1 & Bleeding \\
\hline 3 & 0.97 & 1986 & $3 \mathrm{~d}$ & HLHS & IAT & 3 & 2 & 1 & Bleeding \\
\hline 4 & 2.02 & 2001 & $3 \mathrm{~d}$ & HLHS & EC & 2 & 1 & 1 & Cardiac failure \\
\hline 5 & 1.89 & 1994 & $4 \mathrm{~d}$ & HLHS & $\mathrm{LT}$ & 2 & 1 & 0 & Hypoxemic brain injury \\
\hline 6 & 2.39 & 1987 & $4 \mathrm{~d}$ & HLHS & $\mathrm{LT}$ & 1 & 1 & 1 & Cardiac failure \\
\hline 7 & 1.32 & 1987 & $4 \mathrm{~d}$ & HLHS & $\mathrm{AP}$ & 1 & 1 & 1 & Cardiac failure \\
\hline 8 & 1.33 & 2004 & $6 \mathrm{~d}$ & HLHS & $\mathrm{EC}$ & 3 & 2 & 2 & Bleeding \\
\hline 9 & 1.43 & 1987 & $6 \mathrm{~d}$ & HLHS & $\mathrm{LT}$ & 0 & 0 & 0 & Cardiac failure \\
\hline 10 & 1.58 & 1990 & $10 \mathrm{~d}$ & $\mathrm{TA}$ & $\mathrm{LT}$ & 2 & 1 & 0 & Cardiac failure \\
\hline 11 & 1.92 & 1990 & $20 \mathrm{~d}$ & HLHS & $\mathrm{LT}$ & 3 & 2 & 1 & Empyema \\
\hline 12 & 1.45 & 1994 & $20 \mathrm{~d}$ & HLHS & $\mathrm{LT}$ & 1 & 0 & 0 & Cardiac failure \\
\hline 13 & 0.93 & 1988 & $24 \mathrm{~d}$ & HLHS & $\mathrm{LT}$ & 1 & 1 & 4 & Pneumothorax \\
\hline 14 & 2.38 & 1990 & $30 \mathrm{~d}$ & DORV, MA & $\mathrm{LT}$ & 2 & 1 & 1 & Sepsis \\
\hline 15 & 1.41 & 1988 & $81 \mathrm{~d}$ & HLHS & LT & 1 & 1 & 1 & Cardiac arrest \\
\hline 16 & 2.17 & 1988 & $183 \mathrm{~d}$ & $\mathrm{TA}$ & RA-RV & 4 & 2 & 2 & Cardiac arrest \\
\hline 17 & 2.38 & 1994 & $223 d$ & HLHS & $\mathrm{LT}$ & 3 & 2 & 2 & Cardiac failure \\
\hline 18 & 4.13 & 2003 & $323 d$ & HLHS & $\mathrm{LT}$ & 2 & 1 & 2 & Cardiac arrest \\
\hline 19 & 5.57 & 1999 & $4.0 \mathrm{y}$ & HLHS & $\mathrm{LT}$ & 6 & 4 & 1 & Cardiac failure, sepsis \\
\hline 20 & 15.3 & 2000 & $13.8 \mathrm{y}$ & DILV & IAT & 2 & 1 & 0 & $\begin{array}{l}\text { Cardiac failure, CMV gastritis, } \\
\text { brain hemorrhage }\end{array}$ \\
\hline 21 & 20.1 & 2005 & $16.5 \mathrm{y}$ & TA & $\mathrm{AP}$ & 4 & 3 & 2 & Sepsis \\
\hline 22 & 23.2 & 2002 & $17.5 \mathrm{y}$ & Single RV & LT & 3 & 2 & 1 & Cardiac failure, bleeding \\
\hline
\end{tabular}

HLHS, Hypoplastic left heart syndrome; $L T$, lateral tunnel; $I A T$, intra-atrial tube graft; $E C$, extracardiac; $A P$, atriopulmonary; TA, tricuspid atresia; $D O R V$, double-outlet right ventricle; $M A$, mitral atresia; $R A-R V$, right atrial-right ventricular; $D I L V$, double-inlet left ventricle, $R V$, right ventricle.

One patient demonstrated stage 4 sinusoidal fibrosis (cardiac cirrhosis); this patient died 24 days after lateral tunnel Fontan owing to multiple pneumothoraces. Figure 1, D, shows grade 2 sinusoidal fibrosis in a 4 -year-old boy with hypoplastic left heart syndrome who died of cardiac arrest 11 months after a lateral tunnel Fontan operation.

Portal inflammation was uncommon because $14(64 \%)$ patients had no inflammation, $7(32 \%)$ had mild inflammation, $1(5 \%)$ had moderate inflammation, and no patients had severe inflammation. Of the 7 patients with mild inflammation, 2 died of sepsis and 1 died of cardiac arrest in the setting of gastroenteritis. The patient with moderate inflammation died of an empyema.

For regression analysis, time from Fontan was transformed using the natural log scale because the variable is not normally distributed. After this transformation, time from Fontan was significantly associated with Ishak $(P=.03)$ and modified Scheuer $(P=.02)$ fibrosis stages (Figure 2, $A$ and $B$ ). Time from Fontan was not associated with the severity of sinusoidal fibrosis. Other patient variables including age at Fontan, cardiac anatomy, Fontan type, and presence of fenestration were not associated with Ishak fibrosis stage, modified Scheuer fibrosis stage, or sinusoidal fibrosis stage. Also, the degree of portal fibrosis, by either Ishak or modified Scheuer fibrosis stage, was not significantly associated with the degree of portal inflammation. Analysis with the cumulative logit model confirmed the simple linear regression results.

Further analysis of the patients who died less than a month after the Fontan operation $(\mathrm{n}=14)$ was performed. Table 3 summarizes relevant pre-Fontan characteristics of these patients. By simple linear regression, length of hospitalization after pre-Fontan cardiac operations $(P=.03)$ and mean right atrial pressure at pre-Fontan catheterization $(P=.04)$ were significantly associated with Ishak fibrosis stage (Figure 3, $A$ and $B$ ) in a univariate model. For every 50 days of hospitalization, the Ishak fibrosis stage increased by 1 unit; for every $3-\mathrm{mm} \mathrm{Hg}$ increase in right atrial pressure, the Ishak fibrosis stage increased by 1 unit. Systemic ventricular end-diastolic pressure at pre-Fontan catheterization $(P=.3)$ and pre-Fontan hemoglobin $(P=.8)$ were not significantly associated with degrees of Ishak fibrosis. The degree of sinusoidal fibrosis was not significantly associated with any of the pre-Fontan variables by simple linear regression. Cumulative logit model confirmed linear regression results.

\section{DISCUSSION}

Hepatic fibrosis has emerged as an important sequela of the Fontan operation with potentially serious long-term 

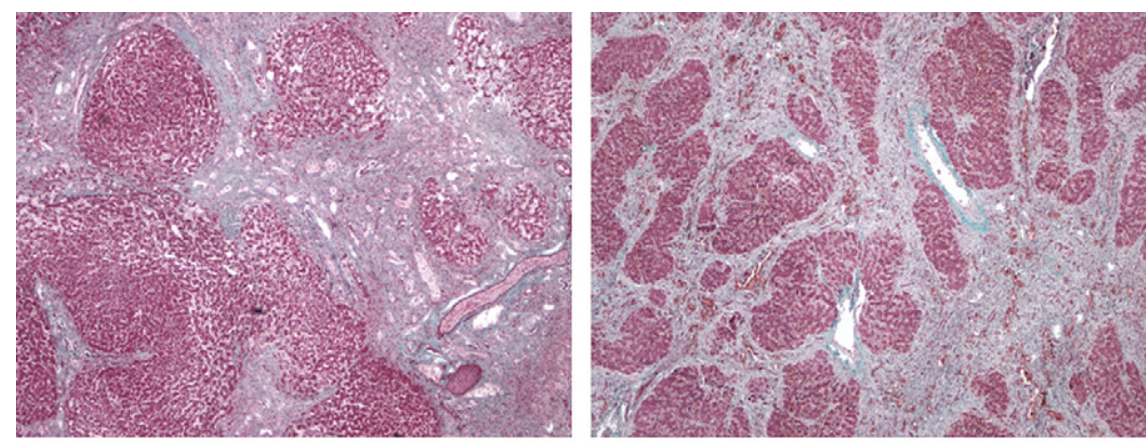

A

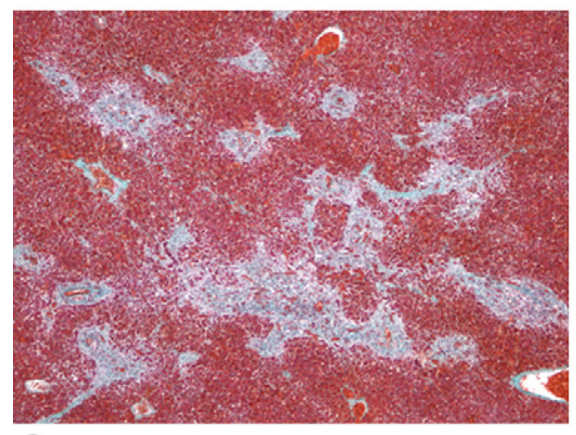

C
B

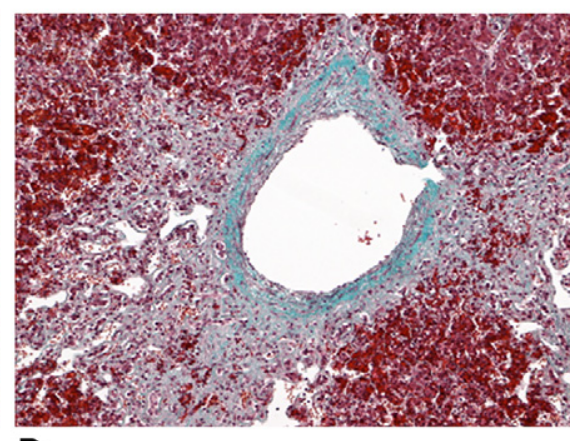

D

FIGURE 1. A, Trichrome stain at $2 \times$ magnification from patient 21 showing significant portal fibrosis in a long-term survivor after the Fontan procedure B, Ttrichrome stain at $4 \times$ magnification from patient 19 showing portal-based cirrhosis 4 years after the Fontan procedure. C, Trichrome stain at $4 \times$ magnification from patient 2 showing significant portal fibrosis only 1 day after the Fontan operation. D, Trichrome stain at $10 \times$ from patient 18 showing sinusoidal fibrosis extending from a central vein 11 months after the Fontan procedure.

morbidity. Until recently, the importance of this complication has not been emphasized. ${ }^{3,4}$ Few descriptions of hepatic histology after Fontan exist and factors associated with the development of fibrosis are poorly defined. ${ }^{6-8}$ Our report is the largest histologic examination of the liver in patients who have undergone the Fontan operation to date and is the first to describe significant portal fibrosis after the operation. It is also the first to describe fibrosis that likely developed before the Fontan procedure.

Previous reports assessing hepatic histology after the Fontan operation have described centrilobular, sinusoidal fibrosis $^{6-8}$; portal-based fibrosis has not been reported. Two of these studies examined the relationship between patient characteristics and histologic findings, suggesting that time from Fontan and central venous pressure elevation are risk factors for centrilobular, sinusoidal fibrosis. ${ }^{6,7}$

In our cohort, most patients showed evidence of both portal and sinusoidal fibrosis. In addition, time from the Fontan operation was associated with portal fibrosis. The etiology of the portal fibrosis in this population is unknown. Portal fibrosis is commonly reported in inflammatory hepatopathies, but it may also be a reflection of elevated central venous pressure and diminished oxygen delivery that characterize the Fontan circulation. However, unique factors such as medication toxicity, genetic abnormalities, or undiagnosed chronic infection such as hepatitis $\mathrm{C}$ may also be responsible for portal-based disease. Data from the United States and Europe suggest that $8 \%$ to $14 \%$ of patients who underwent surgery for congenital heart disease before 1992 are seropositive for hepatitis C antibodies. ${ }^{12,13}$ The median year of surgery in our cohort was 1989, placing many of the patients at risk for hepatitis $\mathrm{C}$ infection. However, 3 of the longest-surviving patients in our cohort had documentation of negative hepatitis $\mathrm{C}$ status. Also, undiagnosed hepatitis $\mathrm{C}$ is unlikely to account for the portal fibrosis observed in patients that died soon after the Fontan operation because of their young age. Portal inflammation was uncommon in our cohort and was not associated with the degree of portal fibrosis.

Sinusoidal liver fibrosis after the Fontan procedure is thought to occur because hepatocytes that surround the central vein experience direct transmission of central venous pressure and are most vulnerable to diminished oxygen delivery. ${ }^{6}$ In our cohort, nearly all of the patients without sinusoidal fibrosis died less than 30 days after the Fontan operation and, thus, had limited exposure to central venous pressure elevation. The degree of sinusoidal fibrosis was not associated with time from Fontan, possibly because there were only 8 patients who died more than 30 days after surgery.

Significant hepatic fibrosis soon after Fontan has not been previously described. At autopsy, 12 of 14 patients who died 

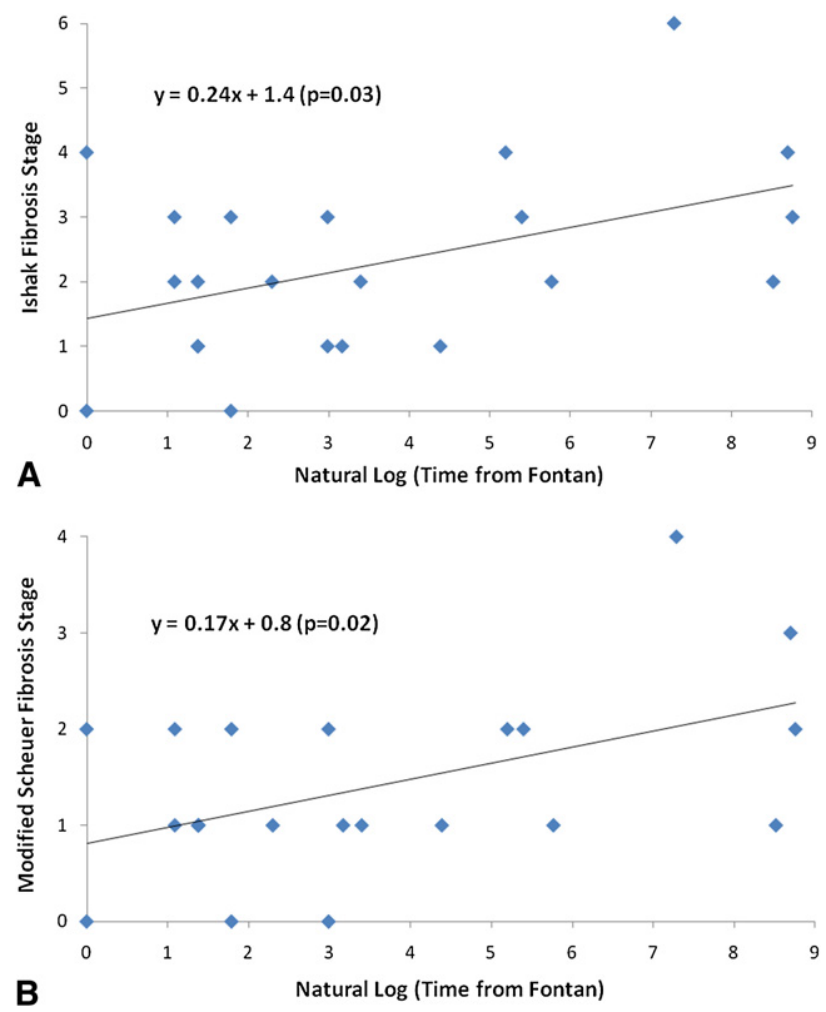

FIGURE 2. A, Simple linear regression analysis of Ishak fibrosis stage and the natural log of time from Fontan interval in 22 patients. B, Simple linear regression analysis of modified Scheuer fibrosis stage and the natural $\log$ of time from Fontan interval in 22 patients.

less than 30 days after the Fontan operation showed portal fibrosis. In many of these patients, the fibrosis was significant. Liver fibrosis is a subacute process and generally takes weeks to months to occur. Thus, it is likely that the fibrosis seen in this subset of patients is a result of pre-Fontan insults. Markers of pre-Fontan morbidity, including length of hospitalization after pre-Fontan cardiac operations and pre-Fontan mean right atrial pressure, were significantly associated with the degree of portal fibrosis among these patients. Prolonged hospitalizations before the Fontan operation with repeated episodes of decreased oxygen delivery might lead to hepatic fibrosis. For example, 1 patient who died 1 day after the Fontan operation had liver

TABLE 3. Pre-Fontan characteristics in the 14 patients who died within 30 days after Fontan operation

\begin{tabular}{lc}
\hline \multicolumn{1}{c}{ Patient characteristic $(\mathbf{n}=\mathbf{1 4})$} & Result* $^{*}$ \\
\hline Hypoplastic left heart syndrome & $12(86 \%)$ \\
No. of Pre-Fontan cardiac operations & $1.5(1-3)$ \\
Cumulative length of hospitalization after & $37.5(10-114)$ \\
$\quad$ pre-Fontan cardiac operations $(\mathrm{d})$ & \\
Pre-Fontan mean right atrial pressure (mm Hg) & $6(3-10)$ \\
Pre-Fontan systemic ventricular end-diastolic & $8(3-12)$ \\
$\quad$ pressure (mm Hg) & \\
*Median (range) or number $(\%)$. &
\end{tabular}
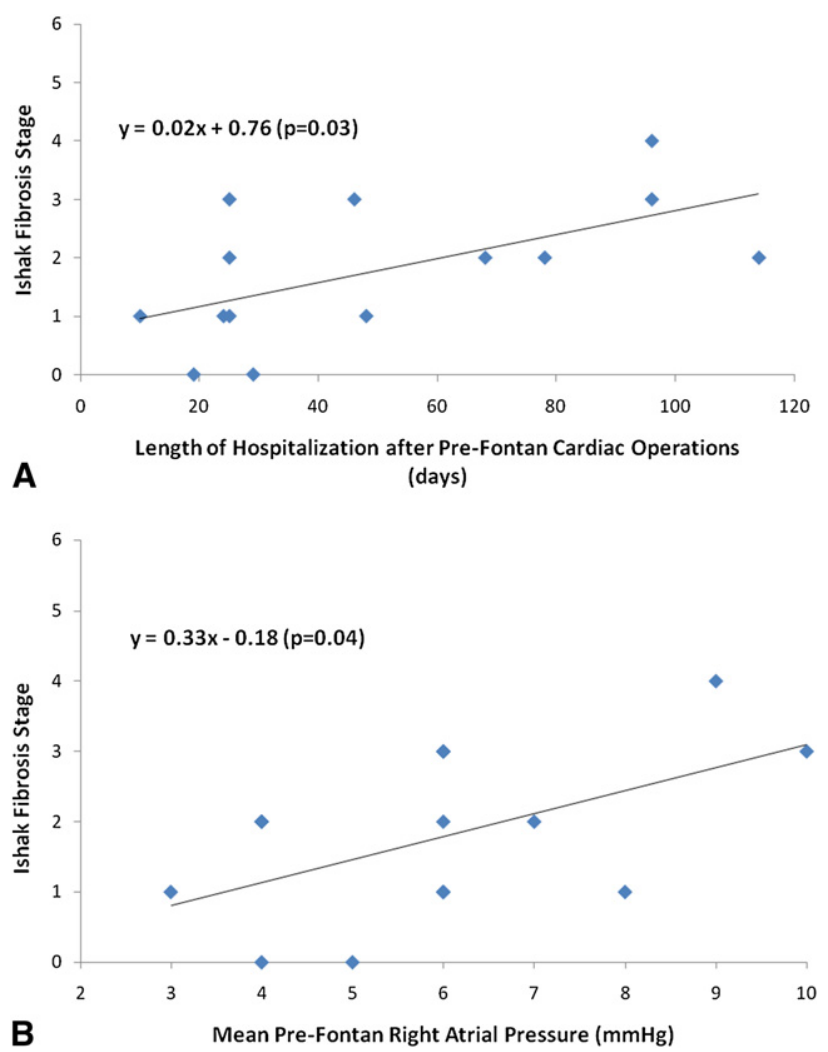

FIGURE 3. A, Simple linear regression analysis of length of hospitalization after pre-Fontan cardiac operations and Ishak fibrosis stage in 14 patients who died 30 days or less after the Fontan operation. B, Simple linear regression analysis of pre-Fontan mean right atrial pressure and Ishak fibrosis stage in 14 patients who died 30 days or less after the Fontan operation.

histology that showed stage 4 Ishak fibrosis. This patient had a protracted hospital course after the Norwood procedure including intubation for 3 months. Mean right atrial pressure before the Fontan operation was also associated with the degree of portal fibrosis in patients who died soon after surgery. Elevated right atrial pressure before the Fontan procedure is likely a surrogate marker of ventricular diastolic dysfunction. Patients with dysfunctional systemic ventricles and higher ventricular end-diastolic pressures before the Fontan procedure may have high hepatic venous pressures and diminished cardiac output, placing them at risk for liver fibrosis.

The etiology of hepatic fibrosis after the Fontan operation is likely multifactorial. We have shown that pre-Fontan morbidity is associated with hepatic portal fibrosis at the time of Fontan and have demonstrated that time from surgery is a risk factor for the development of further portalbased disease. The presence of fibrosis at Fontan may place patients at risk for accelerated progression of disease after surgery. Prospective investigation of liver histology before and after Fontan completion is needed. 


\section{Limitations}

There are several important limitations to our study. The majority of patients in our cohort had hypoplastic left heart syndrome and a history of lateral tunnel, nonfenestrated Fontan. As a result, statistical analysis of possible anatomic and surgical risk factors for hepatic fibrosis was limited. Also, although most patients died of cardiac failure, other causes of death were represented that may have affected hepatic histology. However, fibrosis is a subacute process that is unlikely to develop as a result of acute insults such as infection or cardiopulmonary collapse. We excluded patients with a known cause of liver disease, but extensive serum testing for viral hepatitis was not routinely performed in these patients. Finally, as this was an autopsy examination of hepatic histology, this may be a biased population of Fontan patients and may not be applicable to the general population of children who have had the Fontan operation.

\section{CONCLUSIONS}

At autopsy, portal fibrosis was commonly observed in patients who have undergone the Fontan operation and was associated with time from the operation. Portal-based fibrosis has been previously unrecognized in this population and warrants further investigation into its etiology. Importantly, significant portal fibrosis occurred in the majority of patients who died soon after the Fontan procedure and was associated with pre-Fontan morbidity. These findings suggest that the hepatic disease in the single-ventricle population is multifactorial and may in fact begin before the Fontan operation. As the survival of patients with single-ventricle heart disease continues to improve, the number of adults with Fontan circulation promises to increase. Continued study is needed to understand the timing of liver injury and risk factors for its development and progression.

\section{References}

1. Fontan F, Baudet E. Surgical repair of tricuspid atresia. Thorax. 1971;26:240-8

2. Khairy P, Fernandes SM, Mayer JE Jr. Long-term survivial, modes of death, and predictors of mortality in patients with Fontan surgery. Circulation. 2008;117:85-92.

3. Shah H, Kuehl K, Sherker A. Liver disease after the Fontan procedure: what the hepatologist needs to know. J Clin Gastroenterol. 2010;44:428-31.

4. Wu FM, Ukomadu C, Odze RD, Valente AM, Mayer JE, Earing MG. Liver disease in the patient with Fontan circulation. Congenit Heart Dis. 2011;6:190-201 [Epub 2011 Mar 28].

5. Stanton R, Lurie P, Lindesmith G, Meyer B. The Fontan procedure for tricuspid atresia. Circulation. 1981;64(2 Pt 2):II140-6

6. Ghaferi A, Hutchins G. Progression of liver pathology in patients undergoing the Fontan procedure: chronic passive congestion, cardiac cirrhosis, hepatic adenoma, and hepatocellular carcinoma. J Thorac Cardiovasc Surg. 2005;129:1348-52.

7. Kiesewetter C, Sheron N, Vettukattill J, Hacking N, Stedman B, MillwardSadler H, et al. Hepatic changes in the failing Fontan circulation. Heart. 2007; 93:579-84.

8. Kendall T, Stedman B, Hacking N, Haw M, Vettukattill J, Salmon A, et al. Hepatic fibrosis and cirrhosis in the Fontan circulation: a detailed morphologic review. J Clin Pathol. 2008;61:504-8

9. Ishak K, Baptista A, Bianchi L, Callea F, De Groote J, Gudat F, et al. Histologic grading and staging of chronic hepatitis. J Hepatol. 1995;22:696-9.

10. Lee L, Dai C, Chuang W, Chang W, Hou N, Hsieh M, et al. Comparison of liver histopathology between chronic hepatitis $\mathrm{C}$ patients and chronic hepatitis $\mathrm{B}$ and C-coinfected patients. Hepatology. 2007;22:515-7.

11. Sempoux C, Rahier J. Histological scoring of chronic hepatitis. Acta Gastroen terol Belg. 2004;67:290-3.

12. Wang A, Book WM, McConnel M, Lyle T, Rodby K, Mahle WT. Prevalence of hepatitis $\mathrm{C}$ infection in adult patients who underwent congenital heart surgery prior to 1992. Am J Cardiol. 2007;100:1307-9.

13. Vogt M, Lang T, Frosner G, Klingler C, Sendl AF, Zeller A, et al. Prevalence and clinical outcome of hepatitic $\mathrm{C}$ infection in children who underwent cardiac surgery before the implementation of blood-donor screening. N Engl J Med. 1999; 341:866-70.

APPENDIX 1. Histopathologic grading system used to evaluate hepatic specimen

\begin{tabular}{|c|c|c|}
\hline Feature & Score & Description \\
\hline \multirow[t]{7}{*}{ Ishak portal fibrosis stage } & 0 & No fibrosis \\
\hline & 1 & Fibrous expansion of less than half of portal areas with or without short fibrous septa \\
\hline & 2 & Fibrous expansion of most (more than half) portal areas with or without short fibrous septa \\
\hline & 3 & Fibrous expansion of most portal areas with occasional portal-portal bridging \\
\hline & 4 & Fibrous expansion of most portal areas with marked bridging (portal-portal and portal-central) \\
\hline & 5 & Marked bridging (portal-portal and/or portal-central) with occasional nodules \\
\hline & 6 & Cirrhosis, probable or definite \\
\hline \multirow[t]{5}{*}{ Modified Scheuer portal fibrosis stage } & 0 & No fibrosis \\
\hline & 1 & Enlarged, fibrotic portal tracts \\
\hline & 2 & Periportal or portal-portal septa, but intact architecture \\
\hline & 3 & Fibrosis with distorted architecture but no obvious cirrhosis \\
\hline & 4 & Cirrhosis, probable or definite \\
\hline \multirow[t]{5}{*}{ Sinusoidal fibrosis } & 0 & No fibrosis \\
\hline & 1 & Fibrosis present in less than one thirdof sinusoids \\
\hline & 2 & Fibrosis present in one third to two thirds of sinusoids \\
\hline & 3 & Fibrosis present in more than two thirds of sinusoids \\
\hline & 4 & Cardiac cirrhosis (extensive central vein-central vein bridging) \\
\hline \multirow[t]{4}{*}{ Portal inflammation } & 0 & No inflammation \\
\hline & 1 & Mild inflammation in some or all portal areas \\
\hline & 2 & Moderate inflammation in some or all portal areas \\
\hline & 3 & Severe inflammation in all portal areas \\
\hline
\end{tabular}

